Collection SFN 7 (2007) 199-220

(c) EDP Sciences, Les Ulis

DOI: $10.1051 /$ sfn:2007023

\title{
Diffusion de neutrons sur des surfaces. Application à l'étude de films minces magnétiques
}

\section{F. Ott ${ }^{1}$}

${ }^{1}$ Laboratoire Léon Brillouin, UMR 12 CEA/CNRS, CEA Saclay, 91191 Gif-sur-Yvette, France

\section{INTRODUCTION}

Notre objectif est de caractériser des surfaces et des interfaces à l'échelle nanométrique. Cela correspond à de très petits volumes de matière de l'ordre de quelques microgrammes. C'est pourquoi il est nécessaire de développer des techniques de diffusion de surface. L'utilisation de la réflexion de neutrons en incidence rasante augmente l'interaction des neutrons avec l'échantillon et rend de telles expériences faisables.

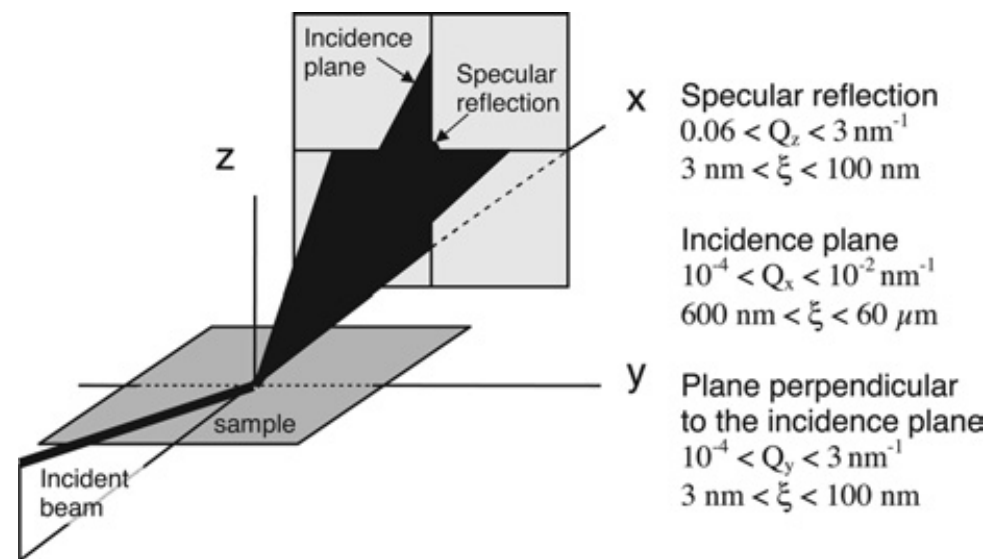

Figure 1. Les différentes géométries de diffusion de surface : (ligne continue) géométrie de réflectivité spéculaire, (surface avec des points) plan de diffusion hors spéculaire, correspondant au plan d'incidence, (surface hachurée) plan de diffusion perpendiculaire au plan d'incidence (GISANS). Ces différentes géométries de diffusion permettent de sonder une très grande gamme de longueurs carac-téristiques et de directions sur la surface de l'échantillon.

Nous allons distinguer trois géométries de diffusion (voir Figure 1) : réflexion spéculaire, diffusion dans le plan d'incidence (diffusion hors spéculaire) et diffusion perpendiculairement au plan d'incidence. Ces différentes géométries de diffusion permettent de sonder différentes longueurs caractéristiques $\xi$ et directions sur la surface de l'échantillon. Dans une première partie nous présenterons les principes de la réflectivité spéculaire qui permet de sonder la structure le long de la profondeur dans le film $(3 \mathrm{~nm}<\xi<100 \mathrm{~nm})$. Nous présenterons le principe de la technique et quelques exemples d'applications sur des systèmes multicouches. Dans une deuxième partie, nous décrirons les principes de la diffusion hors spéculaire qui permet de sonder des détails de la structure planaire à l'échelle micrométrique $(600 \mathrm{~nm}<\xi<60 \mu \mathrm{m})$. Finalement, nous discuterons l'extension de la technique de diffusion aux petits angles pour l'étude des surfaces : GISANS (Grazing Incidence SANS), qui permet de sonder des détails dans la gamme d'échelle $3 \mathrm{~nm}<\xi<100 \mathrm{~nm}$. Ces trois géométries de diffusion permettent de sonder une très large gamme de longueurs caractéristiques $\xi$, allant de quelques $\mathrm{nm}$ à plusieurs $\mu m$. Des structures perpendiculaires et planaires peuvent être sondées. 


\section{RÉFLECTIVITÉ SPÉCULAIRE}

\subsection{Réflexion de neutrons}

Soit $\mathbf{Q}$ le vecteur de diffusion défini par :

$$
\mathbf{Q}=\mathbf{k}_{\mathbf{f}}-\mathbf{k}_{\mathbf{i}}=\frac{4 \pi}{\lambda} \sin \theta
$$

où $\lambda$ est la longueur d'onde du neutron, et $\theta$ est l'angle d'incidence du faisceau de neutrons sur la surface (voir Figure 2). Dans cette géométrie, l'angle de réflexion est égal à l'angle d'incidence ; le vecteur de diffusion $\mathbf{Q}$ est perpendiculaire à la surface de l'échantillon.

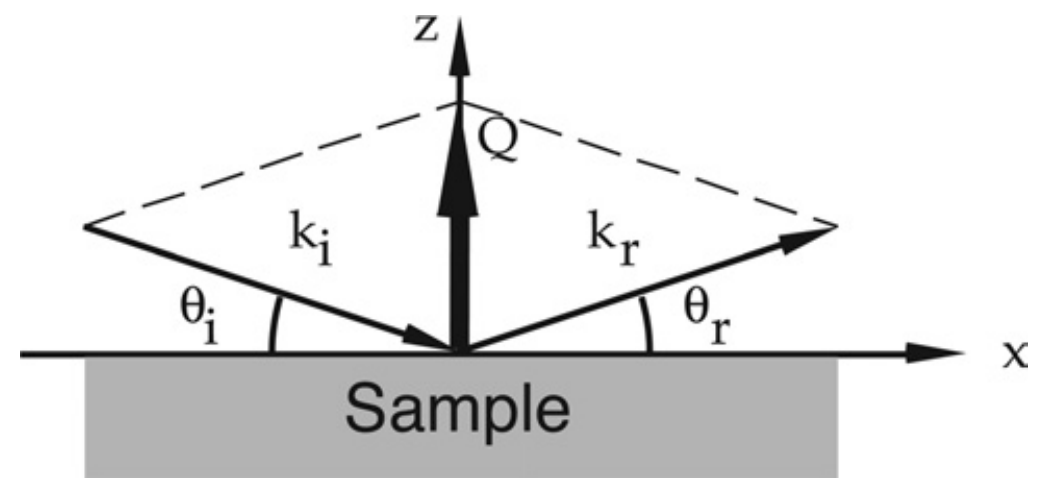

Figure 2. Géométrie de réflexion spéculaire. L'angle de réflexion est égal à l'angle d'incidence ; le vecteur de diffusion $\mathbf{Q}$ est perpendiculaire à la surface de l'échantillon.
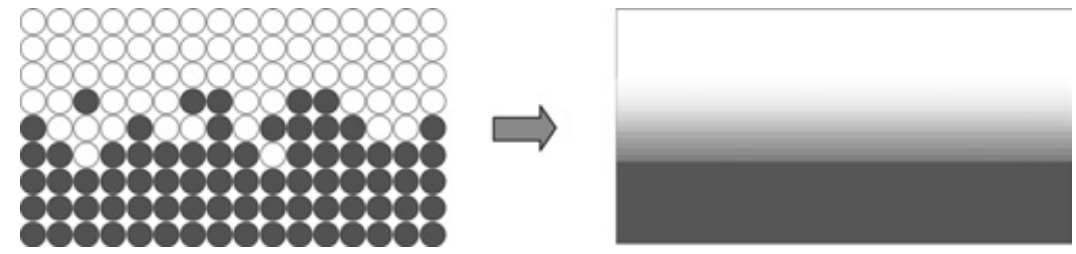

Figure 3. Interface entre deux surfaces. Dans l'approximation optique, l'interface est modélisée par un milieu continu de longueurs de diffusion.

Si on considère une surface, elle est composée d'atomes (voir Figure 3). Cependant, comme les angles d'incidence sont très petits, de l'ordre de quelques degrés, le vecteur de diffusion $\mathbf{Q}$ est de l'ordre de quelques $\mathrm{nm}^{-1}$ (comme déduit de l'Eq. 2.1). C'est-à-dire que l'on sonde des longueurs caractéristiques de l'ordre de quelques nanomètres. Les détails atomiques de la structure sont invisibles. Il est possible d'appliquer l'approximation optique et de considérer le matériau comme un milieu continu. Nous introduisons la densité de longueur de diffusion (SLD Scattering Length Density) donnée par :

$$
S L D=b^{v o l}=\frac{1}{V} \sum_{i \in v o l} b_{i}
$$

où $b_{i}$ est la longueur de diffusion de l'espèce $i$ et $V$ est le volume sur lequel la structure est moyennée (typiquement une cellule atomique). Dans une géométrie de réflectivité spéculaire, on peut faire l'hypothèse que le système est invariant par translation le long de la surface de l'échantillon de telle sorte 
que les contributions de la diffusion proviennent uniquement de la structure le long de l'axe $(O z)$. La Figure 3 illustre la manière dont une interface est modélisée dans une expérience de réflectivité. La longueur de diffusion varie avec la profondeur $z$ dans le film.

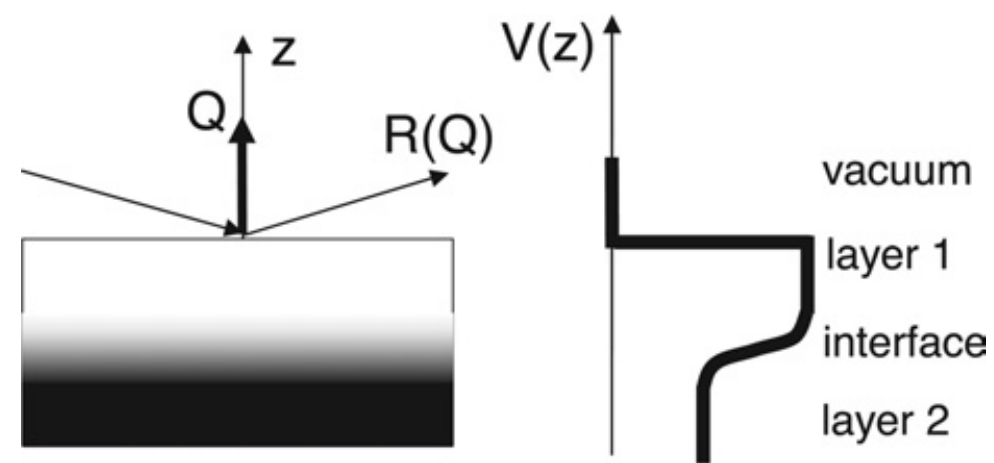

Figure 4. Réflexion sur un film mince déposé sur une surface. La réflectivité mesure la transformée de Fourier du potentiel d'interaction $V(z)$.

\subsection{Quelles informations obtient-on dans une mesure de réflectivité ?}

Le potentiel d'interaction du neutron est donné par :

$$
V(z)=\frac{h^{2}}{2 \pi m_{n}} S L D(z)
$$

où $S L D(z)$ est la densité de longueur de diffusion (Scattering Length Density SLD) le long de la direction $(O z)$.

Soit $R(Q)$ l'intensité réfléchie en fonction du vecteur de diffusion. Dans l'approximation de Born, la réflectivité spéculaire mesure la transformée de Fourier du potentiel d'interaction $V(z)$ le long de la direction perpendiculaire à la surface de l'échantillon :

$$
R(Q) \propto F T(V(z))
$$

Cependant, le faisceau de neutrons peut être totalement réfléchi et alors l'approximation de Born n'est plus valide. Il est alors nécessaire de réaliser un calcul dynamique complet.

\subsection{Calcul de la réflectivité}

Le comportement du neutron est régi par l'équation de Schrödinger :

$$
\frac{-\hbar^{2}}{2 m_{n}} \triangle \varphi+V \varphi=E \varphi
$$

où $\varphi$ est la fonction d'onde du neutron, $E$ est l'énergie cinétique du neutron et $V$ est le potentiel d'interaction (voir Eq. 2.3). Cette équation peut s'exprimer sous la forme d'une équation de propagation de Helmholtz :

$$
\triangle U+k^{2} U=0
$$

où le vecteur d'onde propagation dans le milieu de potentiel $V$ est donné par :

$$
k^{2}=\frac{2 m_{n}}{\hbar^{2}}(E-V)
$$




\section{Indice optique}

Comme les neutrons suivent une équation de propagation classique, il est possible de définir un indice optique $n$ dans le milieu :

$$
n^{2}=\frac{k^{2}}{k_{0}^{2}}
$$

où $k_{0}^{2}$ est le vecteur dans le vide. L'indice optique est donné par :

$$
n^{2}=1-\frac{V}{E}=1-\frac{\lambda^{2}}{\pi} S L D
$$

qui peut être approximé avec une grande précision par :

$$
n \approx 1-\frac{\lambda^{2}}{2 \pi} S L D
$$

A une interface, la réflexion du neutron suit les lois de Snell classiques (voir Figure 5) :

$$
\cos \theta_{i}=n \cos \theta_{t r}
$$

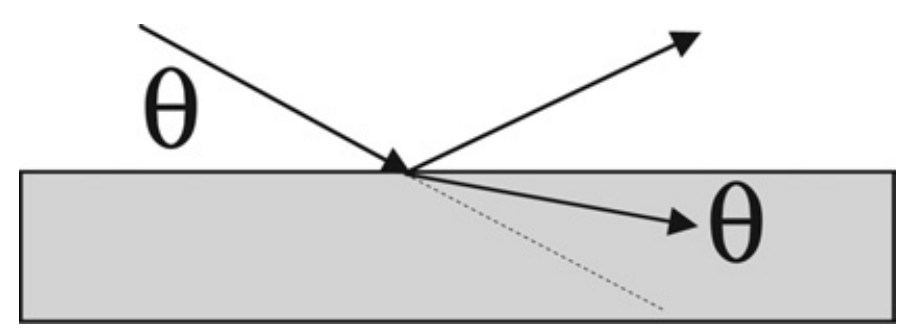

Figure 5. Réfraction d'un faisceau de neutrons à une interface.

Un point important est que l'indice optique est généralement plus petit que 1, cela signifie qu'en dessous d'un angle critique $\theta_{c}$ il y a réflexion totale (définie par la condition $\theta_{t r}=0$ ). L'angle critique est donc donné par l'équation suivante :

$$
n=\cos \theta_{c}
$$

L'expression de l'angle critique est :

$$
\theta_{c}=\sqrt{\pi S L D}
$$

Le vecteur d'onde critique est donné par :

$$
q_{c}=4 \sqrt{\frac{S L D}{\pi} \lambda}
$$

Il est pratique d'exprimer l'indice optique sous la forme :

$$
n \approx 1-\delta=1-\frac{\lambda^{2}}{2 \pi} S L D
$$

où $\delta$ est une petite déviation par rapport à 1 . Il est à noter que l'indice optique dépend de la longueur d'onde et donc la meilleure caractérisation d'un matériau est donnée par la $S L D$. La Table 1 résume les indices optiques des matériaux les plus courants.

On notera que le nickel a l'indice optique le plus élevé de tous les matériaux. Une valeur utile à retenir est que l'angle critique de réflexion totale pour le nickel est de $0.1^{\circ} / \AA$. 
Table 1. Indices optiques de quelques matériaux courants (donnés pour $\lambda=0.4 \mathrm{~nm}$ ).

\begin{tabular}{|l|l|l|}
\hline Elément & $\delta\left(10^{-6}\right)$ & $\theta_{c}\left(^{\circ}\right)$ \\
\hline $\mathrm{Fe}$ & 20.45 & 0.36 \\
$\mathrm{Co}$ & 5.7 & 0.19 \\
$\mathrm{Ni}$ & 24 & 0.4 \\
$\mathrm{Gd}$ & 5.0 & 0.18 \\
$\mathrm{Si}$ & 5.3 & 0.19 \\
$\mathrm{Ti}$ & -5 & - \\
$\mathrm{Al}$ & 5.3 & 0.19 \\
$\mathrm{SiO}$ & 10.1 & 0.26 \\
$\mathrm{H}_{2} \mathrm{O}$ & -1.43 & - \\
$\mathrm{D}_{2} \mathrm{O}$ & 16.2 & 0.33 \\
$\mathrm{H}-$ polystyrène & 3.6 & 0.16 \\
$\mathrm{D}-$ polystyrène & 16.5 & 0.33 \\
\hline
\end{tabular}

\section{Réflexion sur un substrat}

Comme exemple, nous allons dériver le calcul de la réflexion d'un faisceau de neutrons sur le milieu semi-infini.
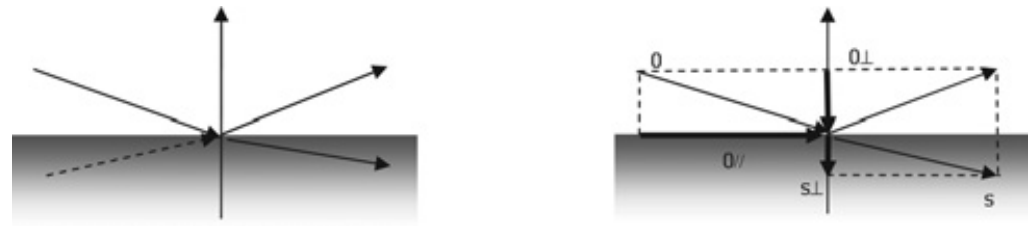

Figure 6. Réflexion d'un faisceau de neutrons sur un milieu semi-infini. Les amplitudes des ondes incidentes, réfléchies et transmises sont respectivement $1, r, t$.

Considérons une onde incidente :

$$
\mathbf{\Psi}(\mathbf{r})=e^{\imath \mathbf{k} \cdot \mathbf{r}}=e^{\imath \mathbf{k}_{\|} \cdot \mathbf{x}} e^{\imath \mathbf{k}_{\perp} \cdot \mathbf{z}}=e^{\imath \mathbf{k}_{\|} \cdot \mathbf{x}} \psi(z)
$$

La composante longitudinale est conservée pendant la réflexion de telle sorte que la fonction d'onde du neutron peut être exprimée comme une fonction uniquement de $z$. Dans chaque milieu, il y a une onde qui se propage vers le haut est une onde qui se propage vers le bas. La fonction d'onde du neutron est exprimée par :

$$
\psi(z)=A \exp \left(\imath k_{\perp} z\right)+B \exp \left(-\imath k_{\perp} z\right)
$$

Dans le vide et dans le substrat nous avons les équations suivantes :

$$
\left\{\begin{array}{l}
\psi_{0}(z)=1 \exp \left(\imath k_{\perp 0} z\right)+r \exp \left(-\imath k_{\perp 0} z\right) \\
\psi_{s}(z)=t \exp \left(\imath k_{\perp s} z\right)+0 \exp \left(-\imath k_{\perp s} z\right)
\end{array}\right.
$$

où 1 est l'amplitude de l'onde incidente, $r$ est l'amplitude de l'onde réfléchie et $t$ est l'amplitude de l'onde transmise (voir Figure 6). Il n'y a pas d'onde provenant de dessous le substrat.

Afin de déterminer les inconnues $r$ et $t$ nous avons besoin de deux équations. Ces équations sont données par les équations de continuité de la fonction d'onde à l'interface $z=0$.

$$
\left\{\begin{array}{l}
\psi_{0}(z=0)=\psi_{s}(z=0) \\
\psi_{0}^{\prime}(z=0)=\psi_{s}^{\prime}(z=0)
\end{array}\right.
$$


qui, en utilisant Eq. 2.18 peut être exprimée comme :

$$
\left\{\begin{array}{c}
1+r=t \\
k_{\perp 0}(1-r)=k_{\perp s} t
\end{array}\right.
$$

Les deux amplitudes $r$ et $t$ peuvent s'exprimer :

$$
\begin{aligned}
r & =\frac{k_{\perp 0}-k_{\perp s}}{k_{\perp 0}+k_{\perp s}} \\
t & =\frac{2 k_{\perp 0}}{k_{\perp 0}+k_{\perp s}}
\end{aligned}
$$

Les signaux de réflectivité mesurés sont les carrés des amplitudes ci-dessus. Le signal physique mesuré est :

$$
R=|r|^{2} \text { and } T=|t|^{2}
$$

Réflectivité sur des systèmes non magnétiques.

La réflectivité sur un substrat peut facilement être calculée. En dessous du vecteur d'onde critique $Q_{c}$, les neutrons sont totalement réfléchis : $r=1$. Approximativement au-dessus de $3 Q_{c}$, la réflectivité décroît comme $1 / Q^{4}$ (en suivant l'approximation de Born classique).

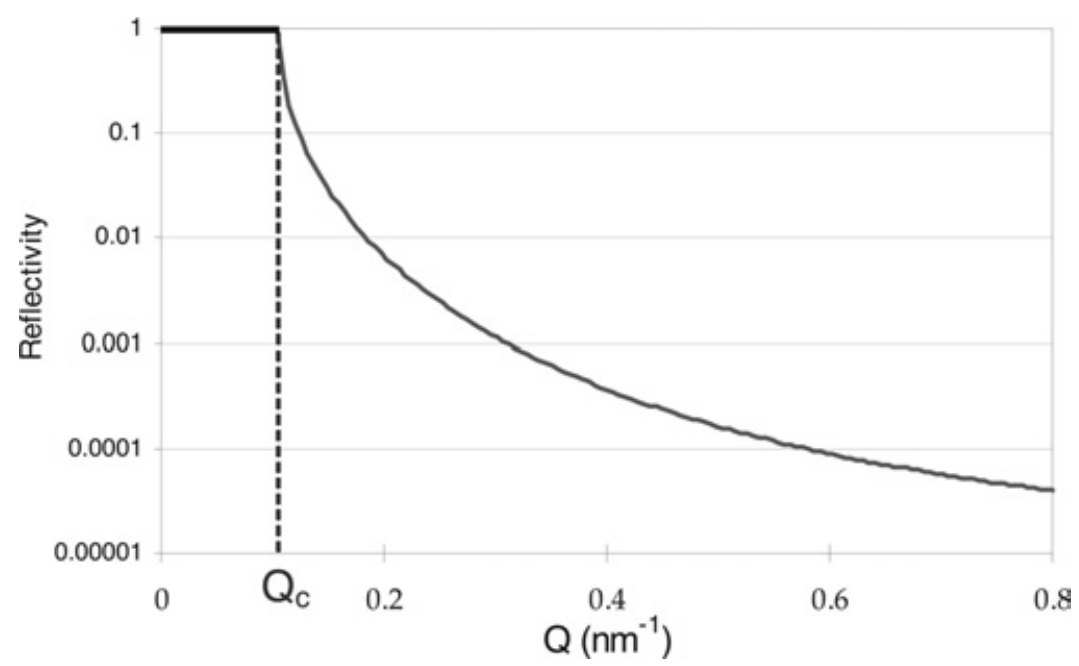

Figure 7. Réflectivité sur un milieu semi- infini, en dessous du vecteur d'onde critique $Q_{c}$, il $y$ a réflexion totale ; au-dessus de ce point, le signal décroît en $1 / Q^{4}$.

Dans le cas d'un film déposé sur un substrat, des oscillations apparaissent dans la courbe de réflectivité. Elles sont dues aux interférences constructives et destructives entre les ondes partiellement réfléchies sur les deux interfaces (voir Figure 8). La période des oscillations correspond à l'épaisseur de la couche $d=2 \pi / \triangle q$. Ces oscillations sont souvent appelées franges de Kiessig.

Lorsque la multicouche est plus compliquée, la figure d'interférence est complexe et un calcul numérique est nécessaire. La Figure 9 illustre le cas d'une couche de cuivre couverte par une fine couche de protection de chrome. La couche de couverture introduit une modulation à longue distance au-dessus des franges d'interférences dues à l'épaisseur de la couche de cuivre. 


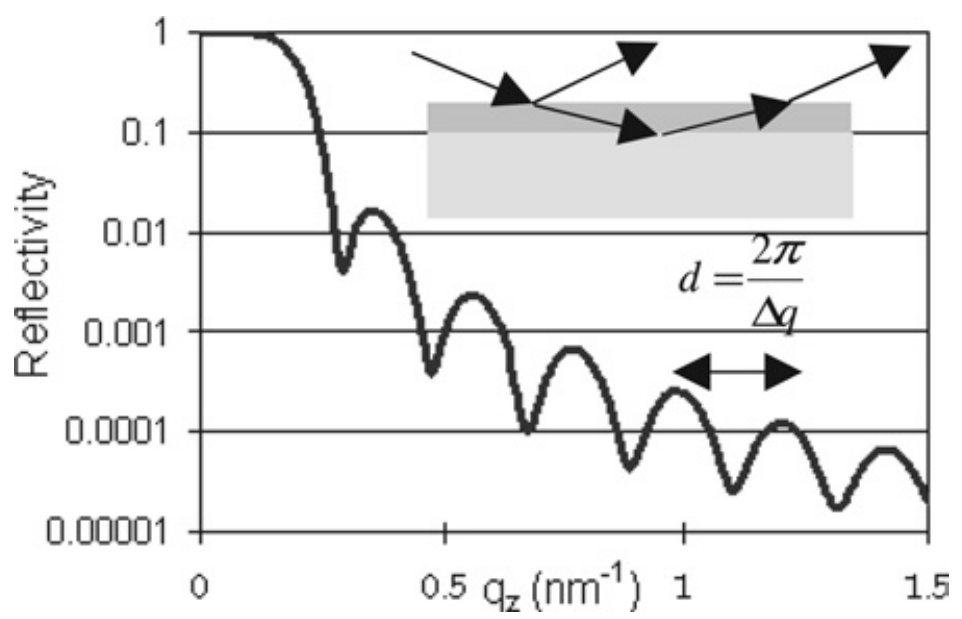

Figure 8. Réflectivité sur un film de cuivre $(30 \mathrm{~nm})$ déposé sur un substrat de silicium.

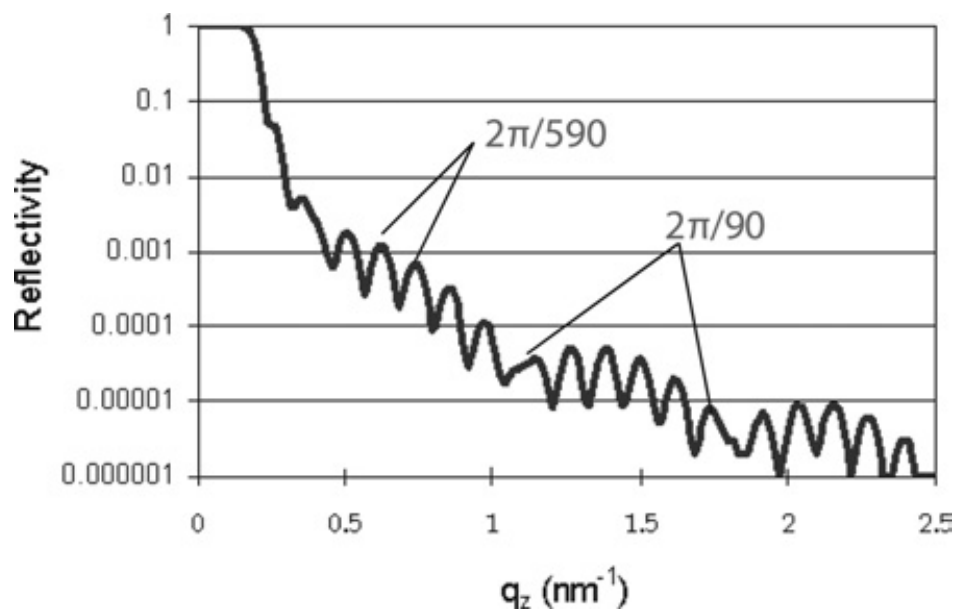

Figure 9. Réflectivité sur un milieu semi infini (substrat de cuivre) et sur un système multicouches $\mathrm{Si} / \mathrm{Cu}$ $(50 \mathrm{~nm}) / \mathrm{Cr}(9 \mathrm{~nm})$. Les oscillations de courte période sont caractéristiques de l'épaisseur totale de la couche $(59 \mathrm{~nm})$; la modulation à longue distance est caractéristique de la fine couche de couverture de chrome $(9 \mathrm{~nm})$. (Insert) profil d'indice optique en fonction de la profondeur dans le film.

\section{RÉFLECTIVITÉ DE NEUTRONS POLARISÉS}

\subsection{Interaction magnétique des neutrons}

Au-delà de l'interaction nucléaire entre le neutron et les noyaux, il y a une interaction directe entre le spin du neutron et l'induction magnétique. Cette interaction est simplement donnée par l'interaction Zeeman :

$$
V_{M}(\mathbf{r})=-\boldsymbol{\mu}_{\boldsymbol{n}} \cdot \mathbf{B}(\mathbf{r})
$$

où $\mu_{n}$ est le moment magnétique du neutron et $\mathbf{B}(\mathbf{r})$ est l'induction magnétique. 
Dans un film mince continu, le champ magnétique dans la couche est donné par :

$$
\mathbf{B}=\mu_{0}\left(\mathbf{H}_{\mathbf{0}}+(1-D) \mathbf{M}\right)
$$

où $\mathbf{H}_{0}$ est le champ magnétique appliqué, $\mathbf{M}$ est l'aimantation du film mince et $D$ est le facteur démagnétisant dans un film mince :

$$
(1-D) \mathbf{M}=\mathbf{M}_{\|}
$$

où $\mathbf{M}_{\|}$est la projection de l'aimantation dans le plan du film. Ceci est une limitation de la PNR : la technique est uniquement sensible à la composante planaire de l'aimantation. L'interaction du neutron avec la matière et donc donnée par :

$$
V=\frac{h^{2}}{2 \pi m} S L D-\boldsymbol{\mu}_{\boldsymbol{n}} \cdot \mu_{0} \mathbf{M}-\boldsymbol{\mu}_{\boldsymbol{n}} \cdot \mathbf{B}_{\mathbf{0}}
$$

Afin de comparer les interactions nucléaires et magnétiques, il est possible d'exprimer le potentiel d'interaction sous la forme :

$$
V=\frac{h^{2}}{2 \pi m} \rho\left(b_{n}+b_{M}\right) \text { avec } b_{M}=\frac{2 \pi m}{h^{2}} \frac{\mu_{n} \mu_{0} M_{\|}}{\rho}
$$

où $\rho$ est la densité atomique.

La Table 2 présente la comparaison entre les longueurs de diffusion nucléaire et magnétique pour les matériaux magnétiques les plus courants. On remarque immédiatement que les deux interactions sont du même ordre de grandeur. Dans le cas du fer, la longueur de diffusion magnétique est de l'ordre de $50 \%$ de la longueur de diffusion nucléaire. Dans le cas du cobalt, la longueur de diffusion magnétique est deux fois plus importante que la longueur de diffusion nucléaire.

Table 2. Comparaison des longueurs de diffusion nucléaire et magnétique pour les matériaux magnétiques usuels.

\begin{tabular}{|l|l|l|}
\hline Elément & $b_{n}(\mathrm{fm})$ & $b_{M}(\mathrm{fm})$ \\
\hline $\mathrm{Fe}$ & 9.45 & 5.4 \\
$\mathrm{Co}$ & 2.49 & 4.5 \\
$\mathrm{Ni}$ & 10.3 & 1.6 \\
$\mathrm{Gd}$ & $6.5-\mathrm{i} 13.82$ & 18.83 \\
$\mathrm{Si}$ & 4.15 & \\
$\mathrm{Ti}$ & -3.44 & \\
\hline
\end{tabular}

\subsection{Réflectivité sur un système magnétique}

Dans la suite, nous appellerons 'up' (resp. ‘down'), les neutrons dont la polarisation est parallèle (resp. antiparallèle) au champ magnétique appliqué qui définit l'axe de quantification. La fonction d'onde du neutron s'exprime sous la forme d'un spineur :

$$
\varphi=a|+\rangle+b|-\rangle=\left(\begin{array}{l}
a \\
b
\end{array}\right) \begin{aligned}
& |+\rangle \\
& |-\rangle
\end{aligned}
$$

Dans le cas d'un film mince magnétique, le système est biréfringent. L'indice optique dépend de la polarisation du neutron par rapport à l'aimantation. L'indice optique s'exprime comme :

$$
n^{ \pm} \approx 1-\delta \pm \delta_{M}=1-\frac{\lambda^{2}}{2 \pi} S L D \pm \frac{m \lambda^{2}}{h^{2}} \boldsymbol{\mu} \cdot \mathbf{B}
$$

où $n^{+}$est l'indice optique pour les neutrons 'up' et $n^{-}$l'indice optique pour les neutrons 'down'. 
Dans une expérience classique de PNR, il est possible de mesurer quatre sections efficaces :

$$
R^{++}, R^{--}, R^{+-}, R^{-+}
$$

- $R^{++}$correspond à l'amplitude réfléchie d'un faisceau de neutrons incident 'up' et à la mesure de la fraction de neutron 'up' après réflexion, c'est-à-dire sans spin-flip pendant la réflexion.

- $R^{+-}$correspond à l'amplitude réfléchie d'un faisceau de neutrons incidents 'up' et à la mesure de la fraction de neutrons "down" réfléchis, c'est-à-dire la fraction du faisceau qui a subi un spin-flip pendant la réflexion.)

La matrice de transfert de l'échantillon est donnée par :

$$
\varphi_{\text {out }}=\left(\begin{array}{cc}
R^{++} & R^{+-} \\
R^{-+} & R^{--}
\end{array}\right) \varphi_{i} n
$$

On peut distinguer deux cas extrêmes.

\section{Aimantation parallèle au champ magnétique appliqué}

Pendant la réflexion, le neutron ne subit pas de spin-flip. Il reste dans son état propre. La matrice de transfert de l'échantillon est de la forme :

$$
\left(\begin{array}{cc}
R^{+} & 0 \\
0 & R^{-}
\end{array}\right)
$$

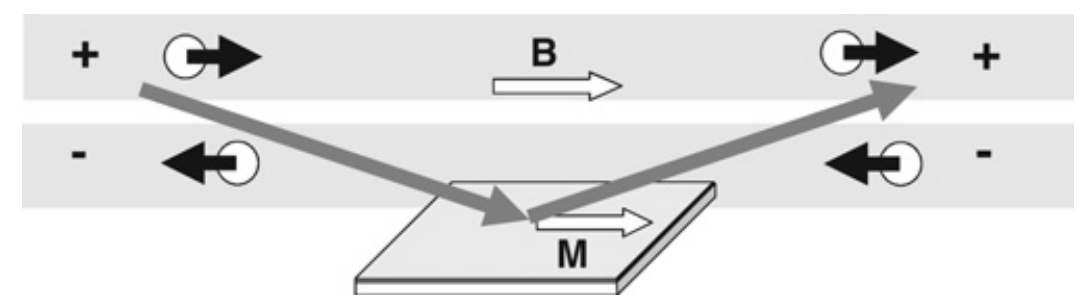

Figure 10. Dans le cas d'une aimantation parallèle au champ magnétique appliqué, il est possible de mesurer 2 sections efficaces différentes $R^{++}$et $R^{--}$.

Pour un faisceau polarisé ‘up' (resp. ‘down'),

$$
\begin{gathered}
\varphi_{\text {in }}=1|+\rangle \quad\left(\text { resp. } \varphi_{\text {in }}=1|-\rangle\right) \\
\varphi_{\text {out }}=\left[\begin{array}{c}
R^{+} \\
0
\end{array}\right]=\left(\begin{array}{cc}
R^{+} & 0 \\
0 & R^{-}
\end{array}\right)\left[\begin{array}{l}
1 \\
0
\end{array}\right] \begin{array}{l}
|+\rangle \\
|-\rangle
\end{array} \text { and }\left[\begin{array}{c}
0 \\
R^{-}
\end{array}\right]=\left(\begin{array}{cc}
R^{+} & 0 \\
0 & R^{-}
\end{array}\right)\left[\begin{array}{l}
0 \\
1
\end{array}\right] \begin{array}{l}
|+\rangle \\
|-\rangle
\end{array}
\end{gathered}
$$

Les amplitudes de réflexion sont données par :

$$
r^{++}=\frac{k_{\perp 0}-k_{\perp s}^{+}}{k_{\perp 0}+k_{\perp s}^{+}} \text {and } r^{--}=\frac{k_{\perp 0}-k_{\perp s}^{-}}{k_{\perp 0}+k_{\perp s}^{-}}
$$

Les intensités réfléchies sont données par :

$$
R^{+}=\left|r^{++}\right|^{2} \text { and } R^{-}=\left|r^{--}\right|^{2}
$$

L'angle critique pour les neutrons 'up' et 'down' est donné par :

$$
n^{+}=\cos \theta_{c}^{+} \text {and } n^{-}=\cos \theta_{c}^{-}
$$



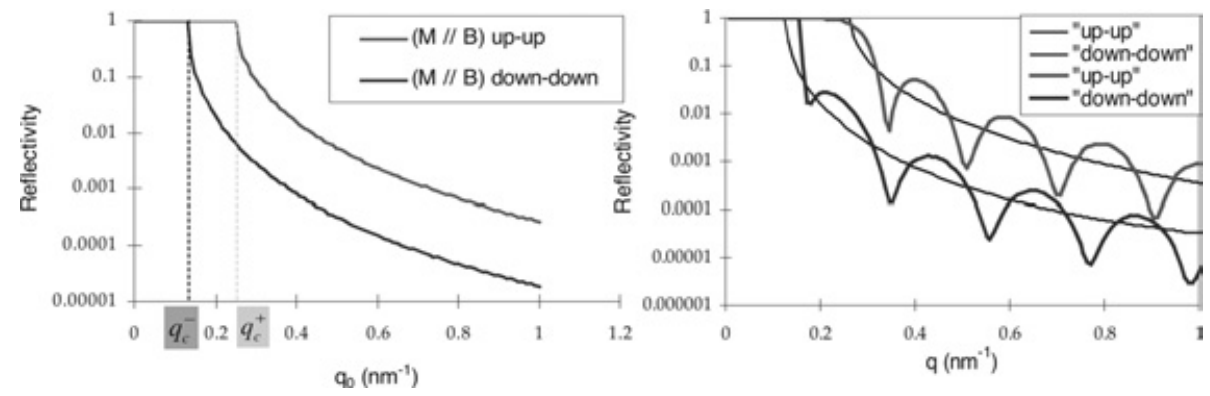

Figure 11. (a) Réflexion sur un milieu magnétique semi-infini ; (b) réflexion sur un film mince magnétique (Fe $(30 \mathrm{~nm})$ sur saphir). Les oscillations sont directement reliées à l'épaisseur du film de fer. La séparation entre les courbes est directement liée à l'aimantation.

La réflexion sur un milieu magnétique semi-infini est très proche du cas magnétique. La seule différence est que les courbes 'up' et 'down' sont distinctes. La différence est directement reliée à l'amplitude de l'aimantation.

\section{Aimantation perpendiculaire au champ magnétique appliqué}

Pendant la réflexion, le neutron va subir un spin-flip partiel, il précesse autour de l'aimantation pendant la réflexion. La matrice de transfert de l'échantillon a la forme générale :

$$
\left(\begin{array}{cc}
R^{++} & R^{+-} \\
R^{-+} & R^{--}
\end{array}\right)
$$

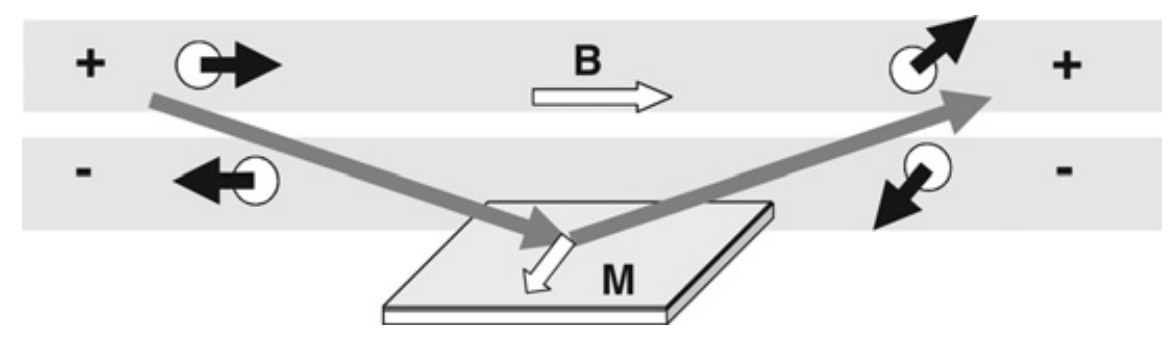

Figure 12. Dans le cas d'une aimantation perpendiculaire au champ magnétique appliqué, les neutrons ne restent pas dans leur état propre. Il est possible de mesurer quatre sections efficaces de diffusion $R^{++}, R^{--}, R^{+-}, R^{-+}$.

Pour un faisceau polarisé 'up' (resp. 'down'), $\varphi_{i n}=1|+\rangle\left(\right.$ resp. $\left.\varphi_{i n}=1|-\rangle\right)$

$$
\begin{aligned}
\varphi_{\text {out }}=\left[\begin{array}{l}
R^{++} \\
R^{+-}
\end{array}\right]= & \left(\begin{array}{ll}
R^{++} & R^{+-} \\
R^{-+} & R^{--}
\end{array}\right)\left[\begin{array}{l}
1 \\
0
\end{array}\right] \mid \begin{array}{l}
|+\rangle \\
|-\rangle
\end{array} \text { and } \\
& {\left[\begin{array}{l}
R^{-+} \\
R^{--}
\end{array}\right]=\left(\begin{array}{ll}
R^{++} & R^{+-} \\
R^{-+} & R^{--}
\end{array}\right)\left[\begin{array}{l}
0 \\
1
\end{array}\right] \mid \begin{array}{l}
|+\rangle \\
|-\rangle
\end{array} }
\end{aligned}
$$

L'intensité de spin-flip réfléchie est donnée par :

$$
r^{+-}=\frac{1}{2} \frac{k_{\perp 0}\left(k_{\perp s}^{+}-k_{\perp s}^{-}\right)}{\left(k_{\perp 0}^{+}+k_{\perp s}^{-}\right)\left(k_{\perp 0}^{+}+k_{\perp s}^{-}\right)}
$$


Dans le cas où l'aimantation est strictement perpendiculaire au champ magnétique appliqué, les intensités réfléchies 'up-up' et 'down-down' sont égales et sont la somme de trois termes :

$$
R^{++}=R^{--}=\left|r^{2}\right|=\frac{1}{4}\left|r^{++}\right|^{2}+\frac{1}{4}\left|r^{--}\right|^{2}+\frac{1}{2} \Re\left(r^{++} \times r^{++}\right)
$$

Les deux premiers termes correspondent aux intensités de non spin-flip dans le cas d'une aimantation alignée avec le champ magnétique extérieur ; elles sont pondérées par un coefficient $1 / 4$. Ces deux termes introduisent 2 discontinuités aux positions $q_{c}^{+}$and $q_{c}^{-}$dans les courbes de réflectivité (voir Figure 13). Un terme d'interférence s'ajoute à cela, dont l'expression n'est pas triviale. Il ne change pas qualitativement la forme des courbes.

L'intensité spin-flip est donnée par :

$$
R^{+-}=R^{-+}=\left|r^{+-}\right|^{2}=\frac{1}{4}\left|\frac{k_{\perp 0}\left(k_{\perp s}^{+}-k_{\perp s}^{-}\right)}{\left(k_{\perp 0}^{+}+k_{\perp s}^{-}\right)\left(k_{\perp 0}^{+}+k_{\perp s}^{-}\right)}\right|^{2}
$$

La forme caractéristique du signal de spin-flip (voir Figure 13) est donnée par le terme $\left|k_{\perp_{s}}^{+}-k_{\perp s}^{-}\right|^{2}$.
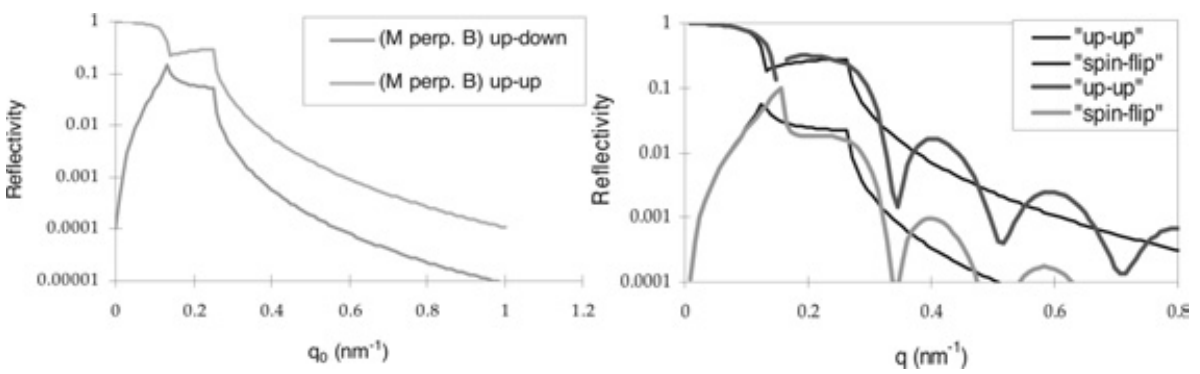

Figure 13. (a) Réflexion sur un milieu magnétique semi-infini où le champ guide est perpendiculaire à l'aimantation; (b) réflexion sur un film mince magnétique $(\mathrm{Fe}(30 \mathrm{~nm})$ sur saphir). Des modulations apparaissent.

Dans une image simple, on peut résumer en disant que les sections efficaces $R^{++}$et $R^{--}$donnent des informations sur les composantes de l'aimantation le long du champ magnétique appliqué. Les sections efficaces de spin-flip $R^{+-}=R^{-+}$donnent des informations sur les composantes de l'aimantation perpendiculaire au champ magnétique appliqué.

\subsection{Instrumentation}

Afin de réaliser une expérience de réflectivité de neutrons polarisés, il est simplement nécessaire d'ajouter quelques éléments optiques sur votre spectromètre deux axes. Vous avez besoin d'un polariseur pour polariser votre faisceau. Le faisceau de neutrons est réfléchi sur l'échantillon et vous utilisez un montage symétrique pour analyser la polarisation réfléchie. De nos jours, les polariseurs et les flippers sont des composants standards peu onéreux.

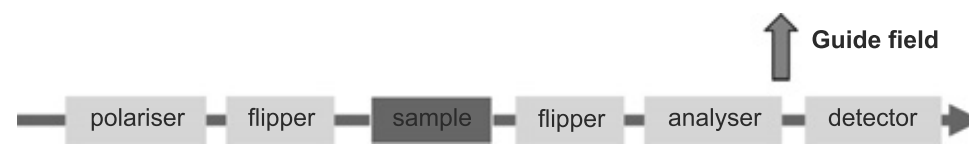

Figure 14. Eléments requis pour réaliser une expérience de neutrons polarisés. 
Actuellement, sur tous les réflectomètres de neutrons polarisés, on travaille avec un petit champ magnétique $\mathbf{B}$ le long du trajet du neutron. Il est possible de mesurer quatre sections efficaces dépendant de la polarisation du neutron par rapport au champ magnétique.
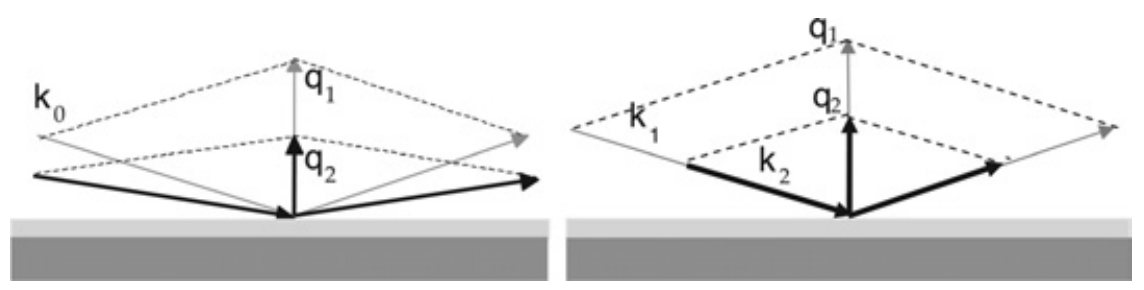

Figure 15. (a) Scan angulaire $\theta / 2 \theta$; (b) scan en temps de vol.

Il y a deux manières de varier le vecteur de diffusion : $Q=4 \pi \sin \theta / \lambda$ :

- Scan angulaire classique $\theta / 2 \theta$. Le réflectomètre utilise une longueur d'onde unique (voir Figure 15(a))

- Temps de vol (Time of Flight ToF) : l'angle d'incidence est fixe et la longueur d'onde varie (typiquement dans la gamme 0.2 à $2 \mathrm{~nm}$ ) (voir Figure 15(b)). Le mode temps de vol est pratique pour l'étude de surfaces liquides libres. Il permet aussi de mesurer la courbe de réflectivité dans son ensemble en une seule fois. Le temps de mesure augmente simplement la statistique.

Le réflectomètre à longueur d'onde fixe est un simple instrument deux axes (voir Figure 16). Le seul besoin est d'avoir de bonnes collimations. Les instruments travaillent typiquement avec une longueur d'onde de 0.4 à $0.5 \mathrm{~nm}$. Les divergences angulaires sont de l'ordre de $0.03^{\circ}(0.5 \mathrm{mrad})$.

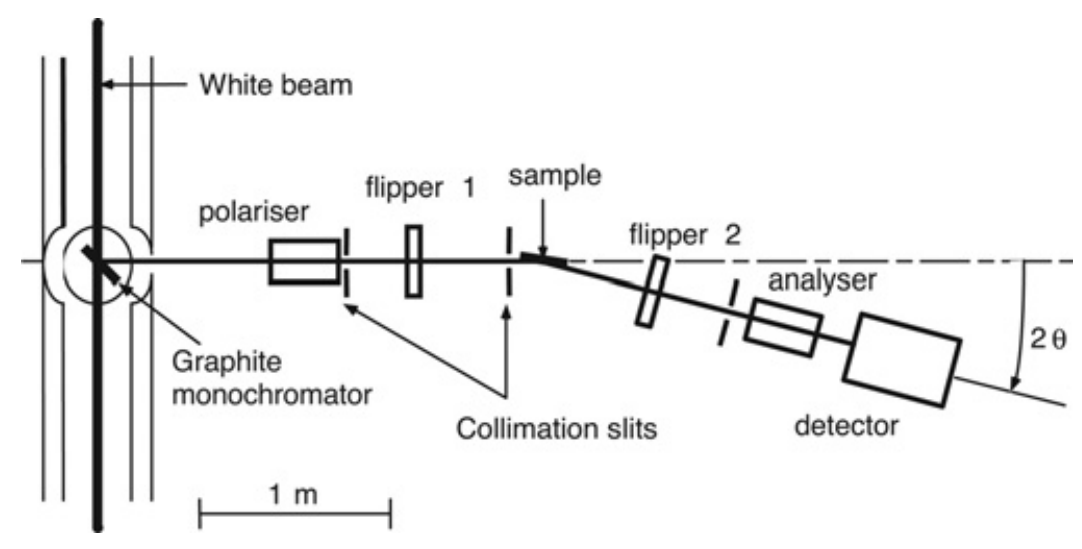

Figure 16. Spectromètre classique deux axes (spectromètre PADA au LLB).

Un réflectomètre en temps de vol est un instrument un peu plus complexe. Il nécessite l'usage d'un chopper sur les sources continue. Dans le cas des neutrons polarisés, un polariseur et un flipper large bande sont nécessaires (voir Figure 17). 


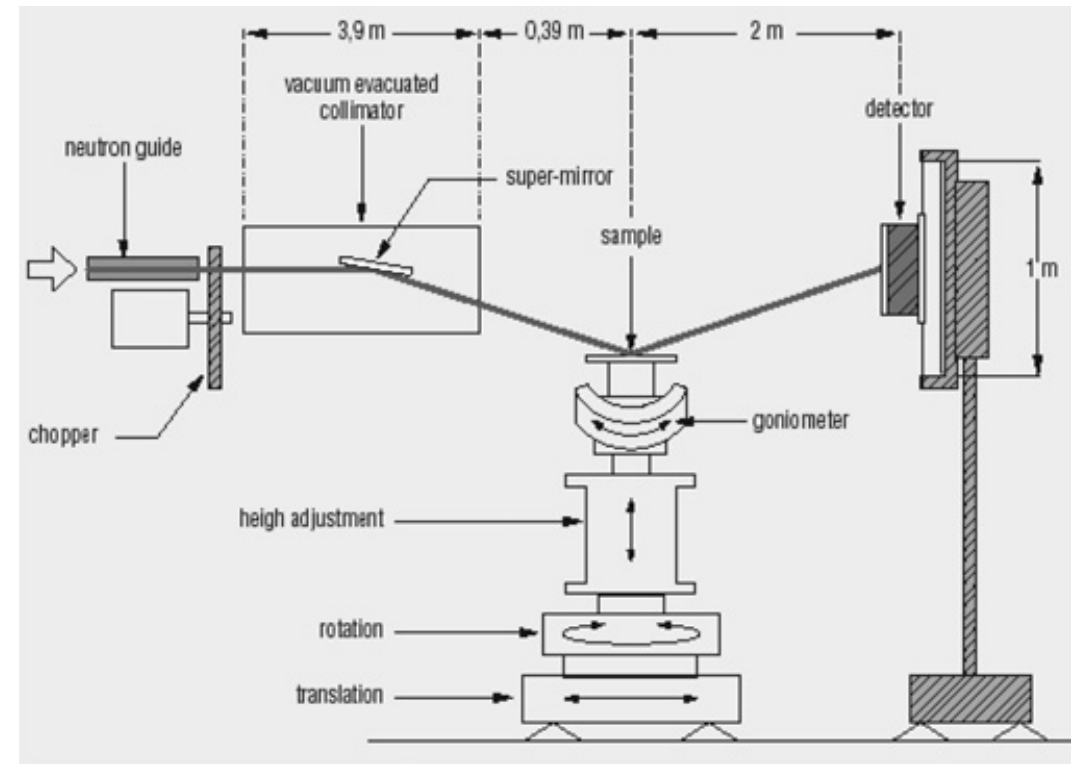

Figure 17. Spectromètre ToF EROS au LLB.

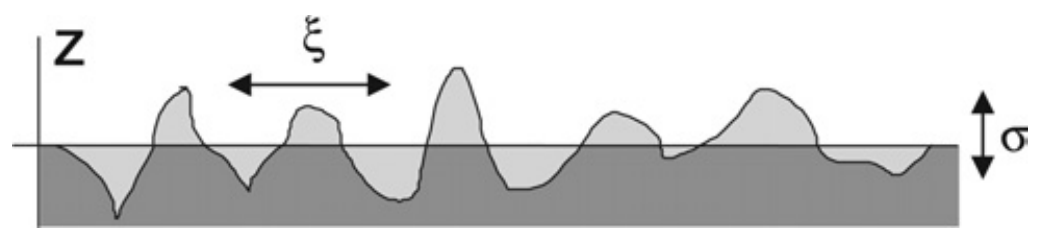

Figure 18. L'interface entre deux matériaux n'est jamais parfaite. La perturbation par rapport à l'interface parfaite et représentée en gris clair.

\section{EFFETS DE RUGOSITÉ}

En réalité, les systèmes étudiés sont toujours imparfaits. Les couches sont composées d'atomes et les interfaces ne sont jamais parfaites. Il est nécessaire de distinguer deux échelles de taille qui sont d'une importance cruciale. La première caractérise les fluctuations de hauteur de l'interface :

$$
\sigma=\sqrt{\left\langle z^{2}\right\rangle_{\text {surface }}}
$$

La seconde longueur caractéristique est donnée par $\xi$ qui caractérise les fluctuations planaires.

En pratique, on peut distinguer quatre types de rugosité :

- Rugosité à l'échelle atomique : elle correspond à l'interdiffusion des atomes à l'interface, typiquement la rugosité $\sigma$ est de l'ordre de $0.5-1 \mathrm{~nm}$ et $\xi<100 \mathrm{~nm}$. Dans ce cas, la réflectivité spéculaire ne peut pas voir ces détails et le système peut être approximé par un profil mou (voir Figure 19(a)).

- Rugosité intermédiaire : $\xi$ de $0.1 \mu m$ to $50 \mu m$ et $\sigma<1 \mathrm{~nm}$. Dans ce cas, une partie du signal diffusé par la surface n'est pas diffusée dans la direction spéculaire. Le signal de réflectivité spéculaire doit être corrigé par un facteur d'atténuation appelé de facteur Névot-Croce $: e^{-\sigma^{2} q^{2}}$. Le signal diffusé hors de la direction spéculaire peut aussi être mesuré et caractérisé. Il donne des 
(a)
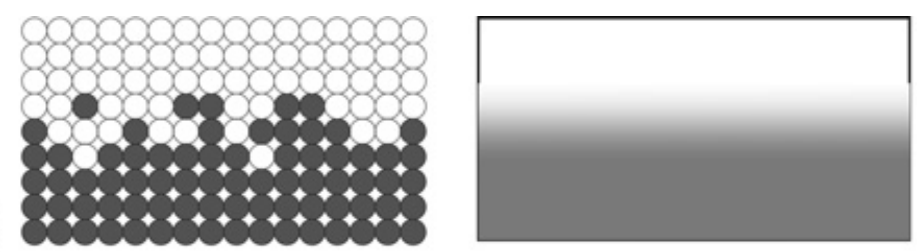

(b)

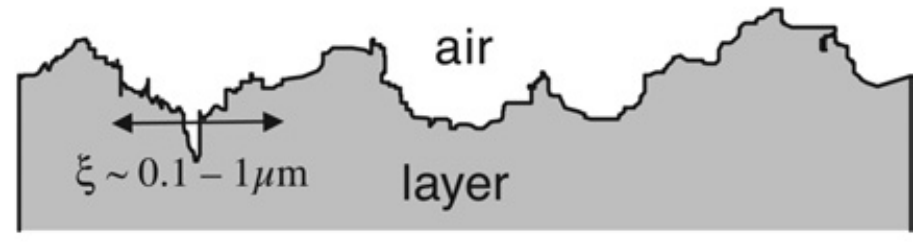

(c)

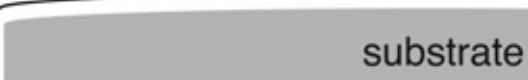

(d)

\section{substrate}

Figure 19. (a) L'interdiffusion atomique entre deux couches ; les détails ne peuvent pas être observés dans une géométrie de réflectivité. (b) la rugosité à l'échelle micrométrique. (c) courbure de l'échantillon. (d) fluctuations de l'épaisseur de la couche.

informations détaillées sur la structure planaire aux interfaces dans le film. Ce thème est traité dans le chapitre sur la diffusion hors spéculaire (E. Kentzinger).

- Rugosité à grande échelle : $\xi>100 \mu \mathrm{m}$. Ces fluctuations correspondent habituellement à un substrat non plan (ou courbure de l'échantillon). Dans ce cas, le moyen le plus simple de traiter les données est de considérer que la divergence incidente ou la résolution en longueur d'onde sont augmentées. Il faut éviter cette situation car l'information de réflectivité est alors fortement altérée.

- Inhomogénéité de l'épaisseur de la couche

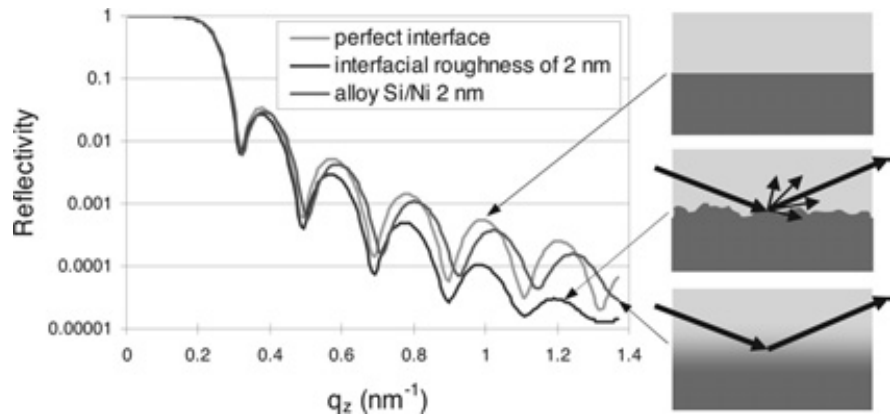

Figure 20. L'influence de la rugosité sur la réflectivité. (i) interface parfaite. (ii) interface rugueuse, des neutrons sont diffusés hors de la direction spéculaire et le signal décroît plus vite aux grandes valeurs de $Q$. (iii) interface douce due à une diffusion atomique, les oscillations sont moins marquées car le contraste d'indice est plus faible. 
Les différents types de rugosité sont comparés sur la Figure 20. Dans le cas de l'interdiffusion atomique (rouge), la forme du signal est très proche du système parfait à l'exception de l'épaisseur effective de la couche supérieure qui est plus faible et qui conduit à un décalage des oscillations. Dans le cas d'une interface rugueuse, des neutrons sont diffusés hors de la direction spéculaire et le signal décroît plus vite à grand vecteur de diffusion $Q$. Il faut appliquer le facteur de Névot-Croce pour rendre compte de cet effet.

\section{EXEMPLES}

Dans ce chapitre, nous allons illustrer les possibilités offertes par la technique de réflectivité de neutrons polarisés. Cette technique peut être appliquée à des systèmes variés :

- films minces : couches d'oxydes (magnétite, manganites)

- multicouches (systèmes à magnéto-résistance géante, GMR)

- super-réseaux : de terres rares (Y, Dy, Er, Tb), semi-conducteurs magnétiques (GaMn-As).
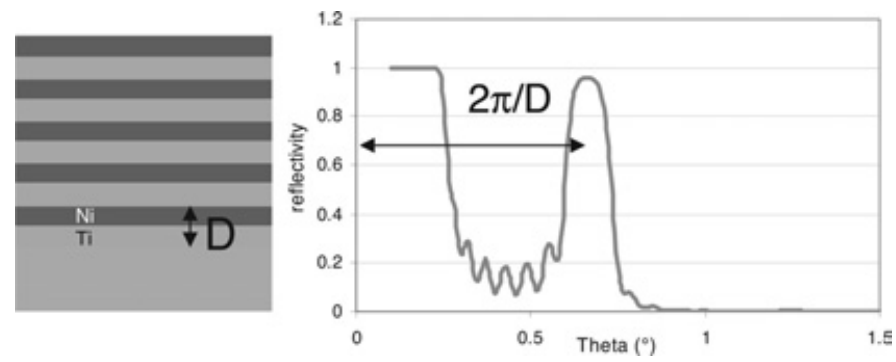

Figure 21. (a) Multicouches $[\mathrm{Ni}(5 \mathrm{~nm}) / \mathrm{Ti}(5 \mathrm{~nm})] 10$ déposées sur du verre. (b) réflectivité de la multicouche. En dessous de l'angle critique, il $y$ a réflexion totale ; autour de $\theta=0.7^{\circ}$, il y a un pic de diffraction correspondant à l'épaisseur de la bicouche $D$. La réflectivité peut être très élevée (proche de $95 \%$ ).

\subsection{Monochromateur multicouches}

Les technologies de films minces permettent de déposer des couches très minces sur des substrats. Il est ainsi possible de produire des cristaux 1D artificiels avec des périodicités aussi faibles que $5 \mathrm{~nm}$ (voir Figure 21). Cela permet notamment de produire des systèmes qui se comportent comme des monochromateurs.
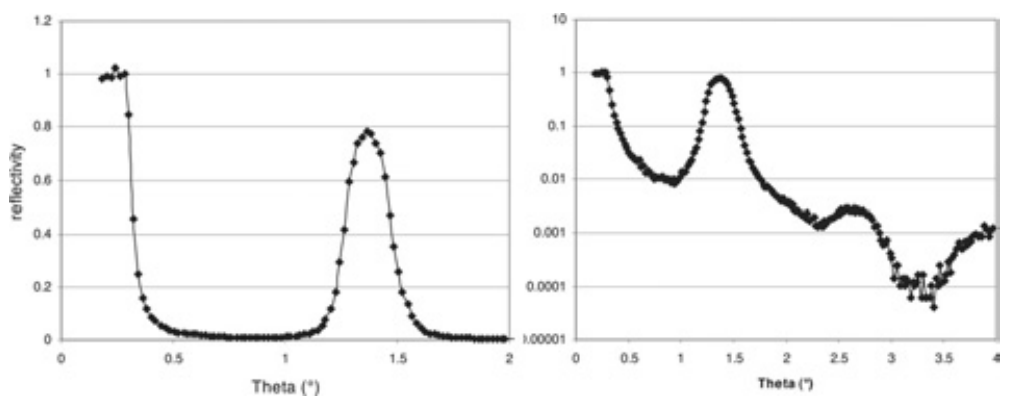

Figure 22. Réflectivité d'un monochromateur produit par Swiss Neutronics (gauche) échelle linéaire ; (droite) échelle logarithmique, les harmoniques de diffraction peuvent être mesurés. 
De telles structures sont maintenant fabriquées de manière routinière sur de très grandes surfaces (de l'ordre du mètre carré) pour produire des monochromateurs de neutrons. La Figure 22 montre la réflectivité d'un monochromateur produit par Swiss Neutronics. La période de la bicouche est de $9 \mathrm{~nm}$. La réflectivité de ce monochromateur atteint $80 \%$. Il est possible d'ajuster la bande de monochromatisation en ajustant la période des bicouches.

Les mesures dans l'espace direct par TEM montrent la qualité des interfaces dans ces multicouches métalliques.

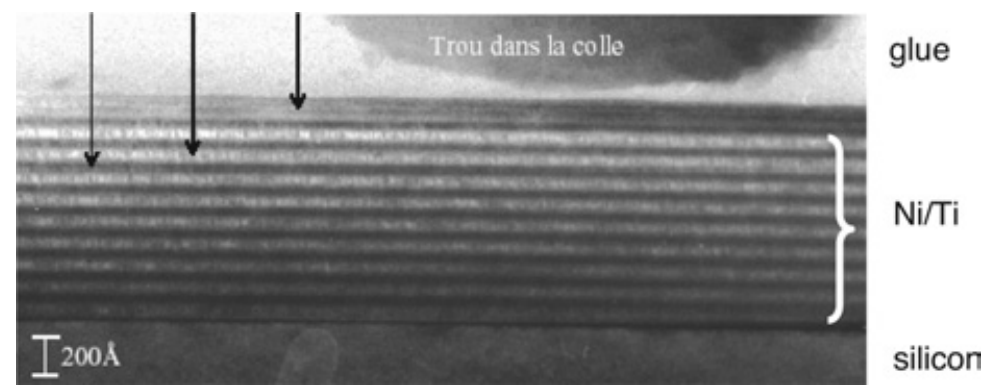

Figure 23. Image HRTEM dans l'espace direct d'un système multicouche.

\subsection{Polariseur de neutrons}

Il est possible d'introduire des matériaux magnétiques dans les systèmes multicouches.

Nota: dans l'exemple précédent, des éléments tels que le carbone sont ajoutés dans le nickel de telle sorte qu'il n'est pas magnétique.

Afin de produire un élément optique efficace, un indice optique élevé est nécessaire. Afin de maximiser le contraste magnétique, les alliages de $\mathrm{Fe}$ ou FeCo sont utilisés. L'espaceur est un matériau proche du silicium afin que son indice optique corresponde à l'indice optique $n^{-}$de la couche magnétique. Quand les neutrons sont polarisés 'up', ils voient une forte modulation de l'indice optique (voir Figure 24(b). Quand les neutrons sont polarisés 'down ', ils voient un potentiel d'interaction lisse et il n’y a pas de diffraction. Un tel dispositif se comporte comme un monochromateur polarisant.
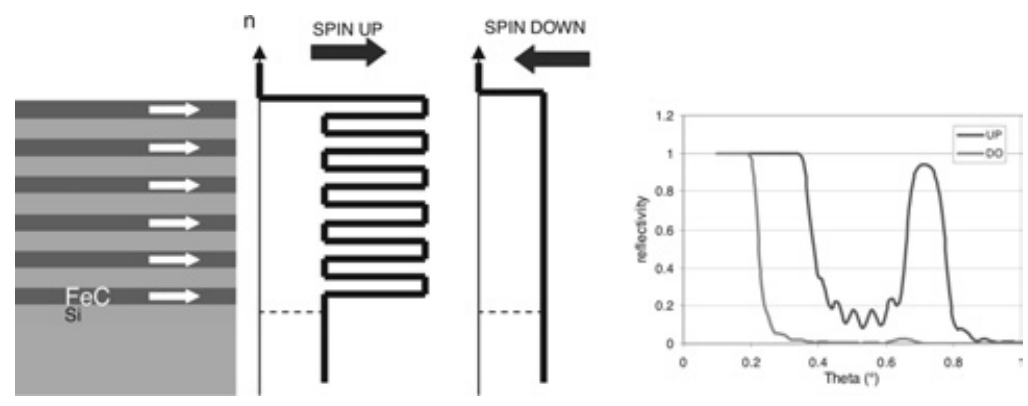

Figure 24. (a) Multicouches [FeCo/Si]10 déposées sur verre. (b) potentiel d'interaction en fonction de la polarisation. (c) réflectivité de la multicouche.

Comme l'absorption des neutrons est très faible, il est possible d'empiler les multicouches de période variable afin de superposer une série de pics de diffraction. Il est ainsi possible de remplir la zone entre la réflexion totale et une valeur $Q_{\max }$ et d'obtenir un miroir d'indice optique artificiel très élevé. La valeur 
$Q_{\max }$ est limitée par les plus petites couches qu'il est possible de déposer (actuellement de l'ordre de $5 \mathrm{~nm}$ ). Cela permet de produire des miroirs qui ont un indice optique artificiel qui est 6 fois plus élevé que celui du nickel.

La Figure 25 montre la réflectivité d'un vrai polariseur (produit par l'ILL). En dessous de $\mathrm{Q}=$ $0.15 \mathrm{~nm}^{-1}$, les deux états de spin se réfléchissent. Au-dessus de $0.15 \mathrm{~nm}^{-1}$, l'état de spin 'down' n'est pas réfléchi ; jusqu'à $0.6 \mathrm{~nm}^{-1}$, l'état de spin 'up' est réfléchi. La Figure 25(b) montre le rapport de flipping up/down, il atteint des valeurs supérieures à 100. Cela correspond à une polarisation du faisceau supérieure à $99.3 \%$.
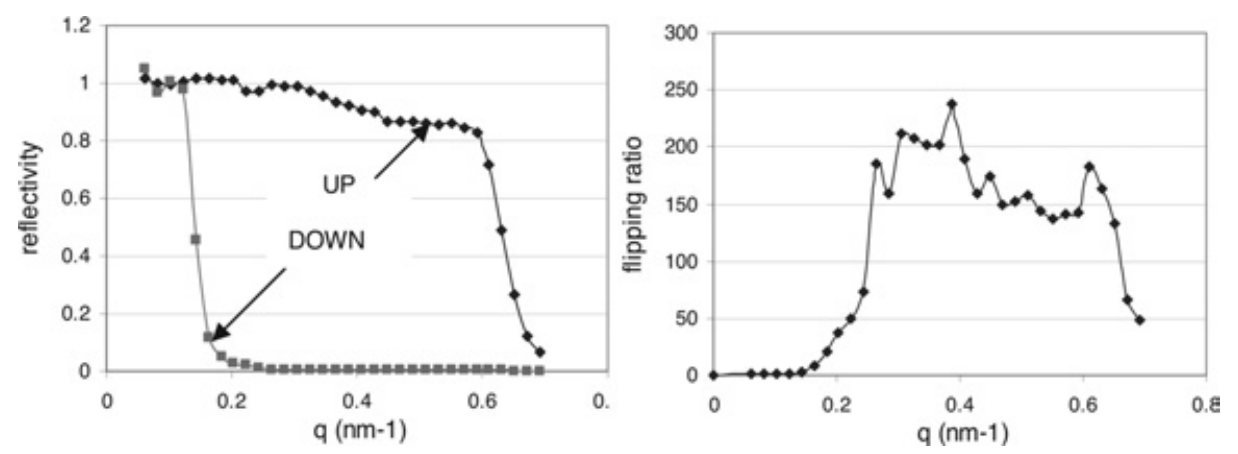

Figure 25. Réflectivité d'un super miroir polarisant.

\subsection{Multicouches magnétiques}

Nous avons présenté des éléments technologiques. De manière plus générale, la réflectivité de neutrons polarisés peut être utilisée pour étudier les configurations magnétiques dans un système multicouches. Cela permet :

- d'accéder à l'amplitude et à la direction de l'aimantation dans chaque couche, c'est-à-dire de déterminer les profils magnétiques en profondeur.

- de mesurer le moment magnétique de manière absolue en magnéton de Bohr.

La résolution en profondeur est de l'ordre de 2 à $3 \mathrm{~nm}$ dans les systèmes simples. La PNR est une technique de surface et n'est donc pas sensible aux contributions paramagnétiques et diamagnétiques du substrat. Il n'y a pas d'absorption. Il n'y a pas de paramètre phénoménologique. Les données sont naturellement normalisées.

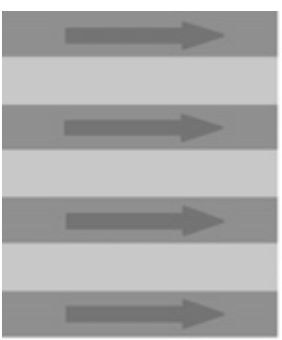

$J_{1}>0$

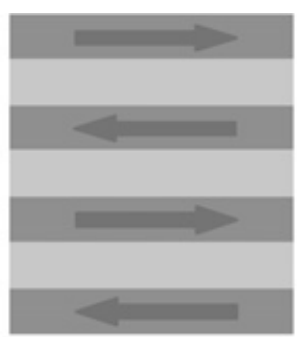

$J_{1}<0$

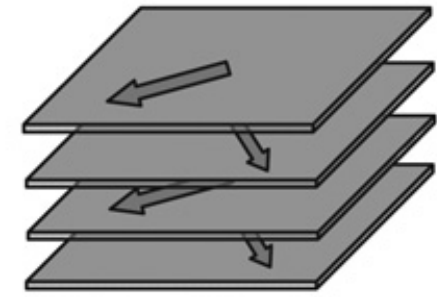

$\mathrm{J}_{1}>0$ and $\mathrm{J}_{2}<0$

Figure 26. (a) Couplage ferromagnétique ; (b) couplage antiferromagnétique (c) couplage quadratique. 


\section{Couplage magnétique}

Dans un système de multicouches magnétiques ù les couches magnétiques sont séparées par un espaceur magnétique, différents types de couplage peuvent apparaître. L'énergie de couplage magnétique peut être décrite phénoménologiquement par une énergie :

$$
E_{\text {coupling }}=-J_{1} \mathbf{S}_{\mathbf{1}} \cdot \mathbf{S}_{\mathbf{2}}-J_{2}\left(\mathbf{S}_{\mathbf{1}} \cdot \mathbf{S}_{\mathbf{2}}\right)^{2}
$$

Couplage d'échange dans les multicouches

Nous présentons l'exemple de multicouches $[\mathrm{Fe} / \mathrm{Si}]_{n}$ produites par K. Fronc. Le système présenté est $\mathrm{GaAs} /[\mathrm{Fe}(2.4 \mathrm{~nm}) \mathrm{Si}(1.2 \mathrm{~nm})]_{20}$. De la mesure de PNR, il est possible de déterminer qu'à température ambiante, un ordre antiferromagnétique apparaît. Quant la température diminue, un ordre magnétique complexe apparait. Sur la mesure de réflectivité (voir Figure 27), on peut observer différents détails. Le premier est le pic (001) de la structure du super réseau qui correspond à la structure chimique du système. Le deuxième détail très important est le pic observé à la position $(001 / 2)$. Ce pic est purement d'origine magnétique. Il indique qu'un couplage antiferromagnétique existe entre les couches de fer.

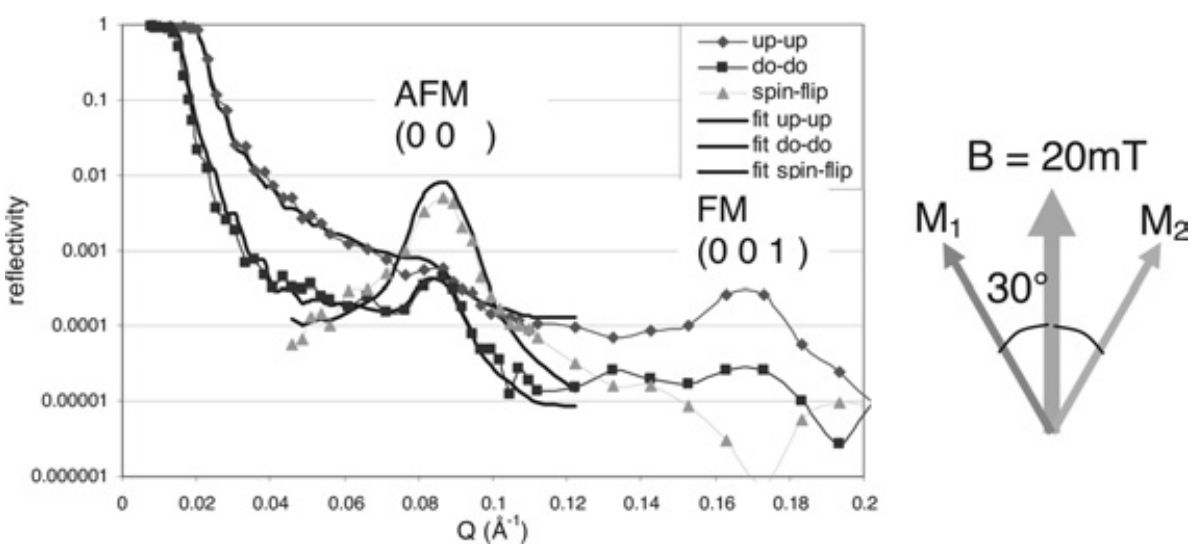

Figure 27. Réflectivité de $[\mathrm{Fe}(2.4) / \mathrm{Si}(1.2)]_{n}$ mesurée à $5 \mathrm{~K}$.

Lorsque la température diminue en dessous de $200 \mathrm{~K}$, à la position du pic antiferromagnétique, un pic de spin-flip devient dominant. Cela signifie que le couplage entre les couches n'est plus purement antiferromagnétique. Un modèle numérique suggère que l'aimantation des couches de fer fait un angle de $30^{\circ}$ par rapport au champ magnétique appliqué.
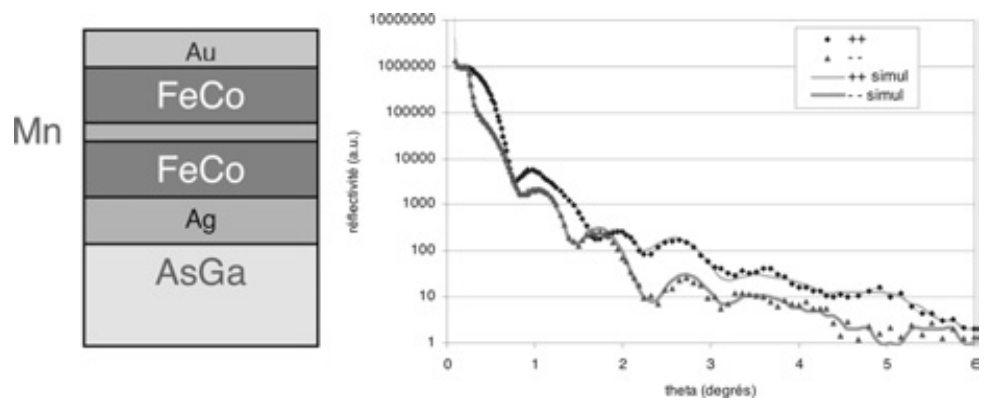

Figure 28. (a) Système tri-couche $\mathrm{Fe} / \mathrm{Mn} / \mathrm{Fe}$; (b) réflectivité dans l'état saturé. 
Couche mince magnétique simple

La PNR permet de sonder les systèmes très fins. Le comportement magnétique de $\mathrm{Fe} / \mathrm{Mn} / \mathrm{Fe}$ a été étudié. La structure typique de l'échantillon est présentée sur la Figure 28(a). La région active est formée par les couches $\mathrm{Fe} / \mathrm{Mn} / \mathrm{Fe}$. La couche d'argent est utilisée pour promouvoir la croissance épitaxiale des systèmes. La couche d'or est une simple couche de protection. Le système présenté est $\mathrm{Fe}_{0.5} \mathrm{Co}_{0.5} / \mathrm{Mn}(8 \AA) /$ $\mathrm{Fe}_{0.5} \mathrm{Co}_{0.5}$. La spécificité de ce système est que les couplages magnétiques entre $\mathrm{Fe} / \mathrm{Mn}$ et $\mathrm{Co} / \mathrm{Mn}$ sont de signes opposés. Cela conduit un comportement magnétique complexe de la couche de manganèse.
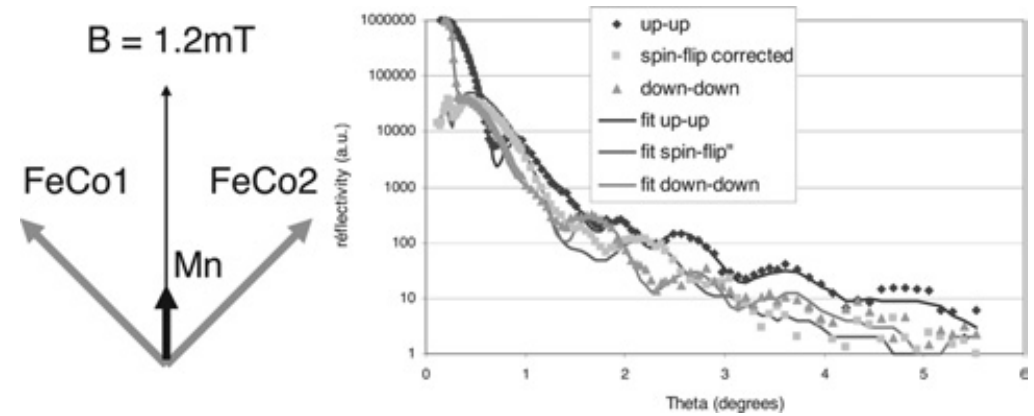

Figure 29. (a) Réflectivité dans l'état rémanent (b) configuration magnétique déduite du fit.

Une première mesure a été réalisée dans un champ saturant (voir Figure 29). Un modèle numérique des données montre que le moment magnétique dans le $\mathrm{Fe}_{0.5} \mathrm{Co}_{0.5}$ est de $2.4 \mu_{B}$ /atome (comme dans le matériau massif). Un moment magnétique net de $0.8 \mu_{B}$ /atome dans le Mn est aussi observé. Une telle aimantation induite a été théoriquement prédite pour les alliages FeCo. Dans des systèmes similaires sans cobalt, aucun moment magnétique n'est observé dans la couche de manganèse.

Le champ magnétique appliqué a été diminué à $1.2 \mathrm{mT}$. La réflectivité a été mesurée à nouveau. Dans ces conditions, un fort signal de spin-flip est observé (voir Figure 29(a)). Les données de réflectivité ont été ajustées en laissant varier la direction de l'aimantation. Le meilleur fit est obtenu lorsque l'aimantation des couches fait un angle de $45^{\circ}$ par rapport au champ magnétique appliqué. Les deux aimantations de la multicouche font un angle de $90^{\circ}$, nous avons un couplage quadratique.

\section{Capteurs GMR}

Dans les 15 dernières années, un gros effort a été dédié à la fabrication et l'optimisation des capteurs à magnéto résistance géante (Giant Magneto-Resistive Sensor GMR) qui consiste en un système magnétique tri-couches : deux couches magnétiques (permalloy) sont séparées par un espaceur non magnétique (cuivre). De tels capteurs sont utilisés dans l'enregistrement magnétique parce que la résistance électrique dépend de l'orientation relative des 2 couches de permalloy. Une couche d'accrochage qui est habituellement un antiferromagnétique tel que FeMn bloque l'aimantation de la couche supérieure de permalloy. L'aimantation de la deuxième couche de permalloy peut tourner librement dans le plan de la couche. La résistivité d'un tel système dépend de l'orientation relative des deux couches magnétiques. En suivant la résistivité, il est possible de mesurer le champ magnétique appliqué et d'utiliser ces systèmes comme des capteurs de champs magnétiques très sensibles.

Un système GMR typique est présenté sur la Figure 30, $\mathrm{SiO} 2 / / \mathrm{Ta} / \mathrm{NiFe} / \mathrm{CoFe} / \mathrm{Cu} / \mathrm{CoFe} / \mathrm{MnPt} /$ Ta. Cette structure est utilisée pour des têtes de lecture dans l'enregistrement sur bande à haute densité. L'objectif de cette étude était de comprendre et d'optimiser le comportement magnétique des différentes couches pour obtenir un système avec un bruit magnétique aussi faible que possible, de telle sorte que le système soit plus performant. 


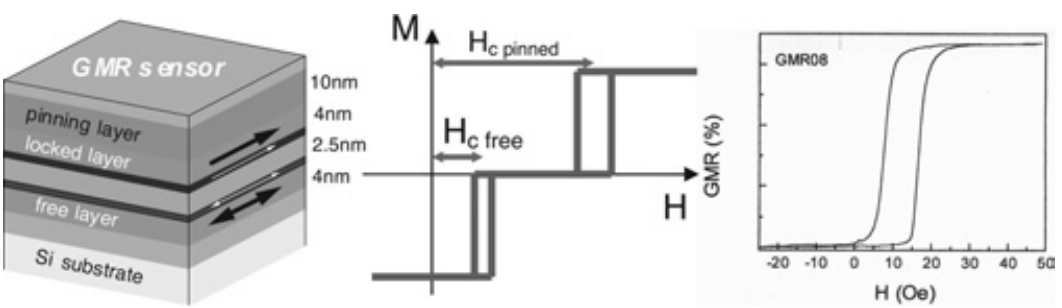

Figure 30. (a) Système GMR typique ; (b) cycle d'hystérésis sur le système ; (c) résistivité du système GMR.

La PNR peut être utilisée pour suivre le comportement des différentes couches du capteur en fonction du champ magnétique appliqué. La Figure 31 montre des mesures d'aimantation sur le système GMR. L'axe facile de la couche antiferromagnétique est perpendiculaire à l'axe facile de la couche libre.

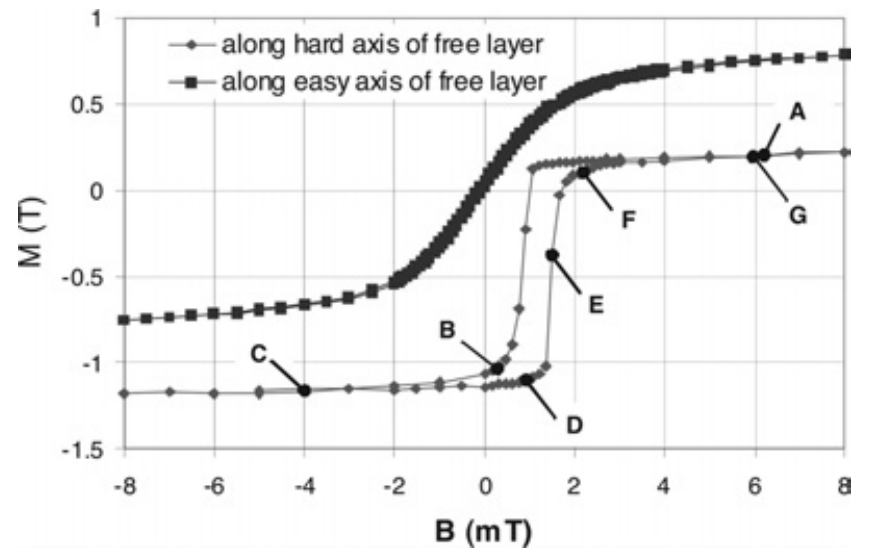

Figure 31. Cycle d'hystérésis de la multicouche.

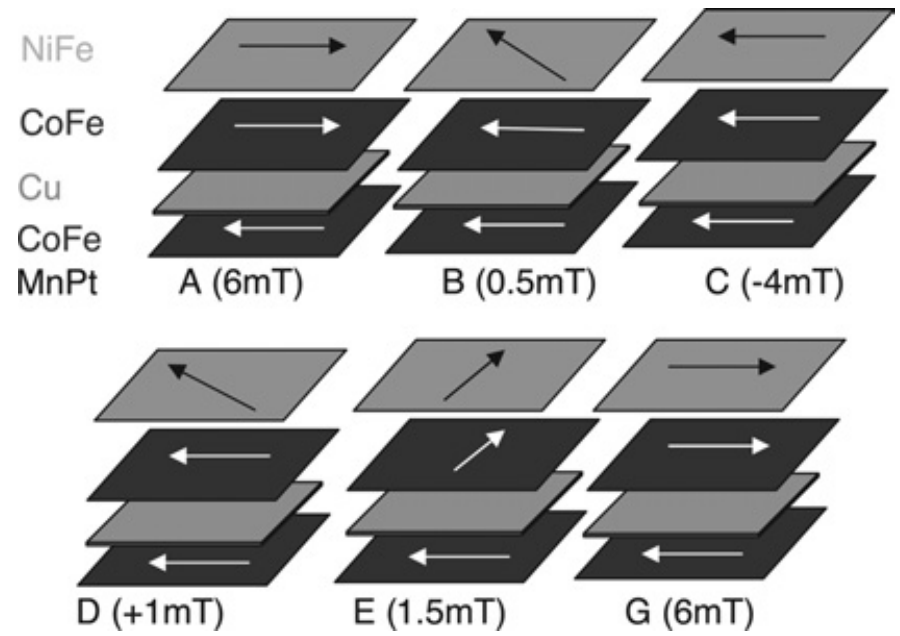

Figure 32. Évolution de la configuration magnétique du système GMR en fonction du champ magnétique appliqué. 
La PNR a été suivie en fonction du champ magnétique appliqué (voir les points sur la Figure 31). La configuration magnétique peut alors être suivie le long du cycle d'hystérésis et il est possible de reconstruire le processus de renversement des couches magnétiques, en particu-lier celui de la couche magnétique douce. Partant de la configuration parallèle, juste après le premier renversement, lorsque le champ diminue, on peut observer que l'aimantation de la couche de permalloy n'a pas complètement flippé mais qu'il $y$ a en fait un gradient de rotation à l'intérieur de la couche de permalloy. D'un côté la couche de permalloy une contrainte par une fine couche de CoFe mais il $y$ a compétition avec l'anisotropie magnétique qui a été introduite pendant le process de dépôt et qui est à $90^{\circ}$ par rapport à cette direction. Le gradient de rotation dans la couche de permalloy atteint $25^{\circ}$ du dessus au-dessous de la couche. Cette rotation est très importante parce que c'est la raison pour laquelle la rotation de l'aimantation des différentes couches est cohérente, c'est-à-dire qu'il n'y a pas formation de domaines magnétiques. Cela est particulièrement important en termes de bruit magnétique dans le système. Sur le chemin retour, on observe le même phénomène.

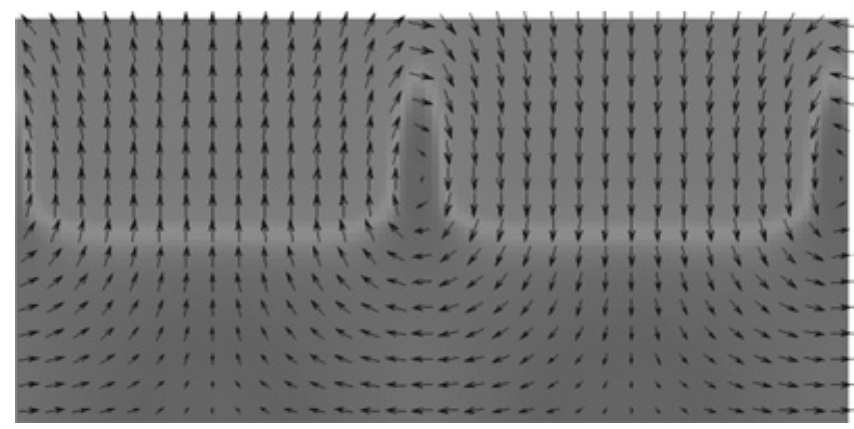

Figure 33. Configuration micro magnétique dans FePd(30 nm)/FePd(30 nm) (vue de côté). La couche de FePd avec une anisotropie perpendiculaire induit la formation de domaines magnétiques.

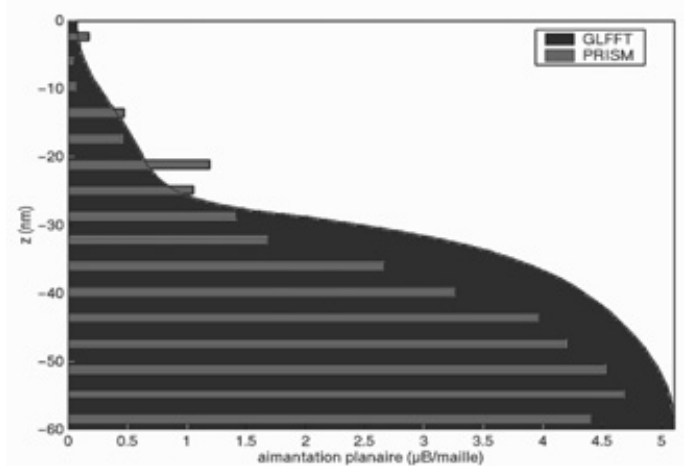

Figure 34. Comparaison entre le profil magnétique déduit du calcul micro magnétique et des data de réflectivité de neutrons. L'accord est très satisfaisant.

\section{Domaines magnétiques}

Par PNR, il est possible de caractériser des profils continus. Pour les couches de FePd, une structure magnétique complexe apparaît. G. Beutier et al du CEA/DRFMC Grenoble ont étudié des systèmes de type bicouche $\mathrm{FePd} / \mathrm{FePd}$ dans lesquels une couche a une anisotropie planaire et la seconde couche a une anisotropie perpendiculaire. 
En utilisant la PNR, il est possible de reconstruire le profil d'aimantation à travers l'épaisseur du système (voir Figure 34). La mesure de PNR ne fournit qu'une information sur l'aimantation planaire moyenne en fonction de la profondeur dans le film. L'accord entre le calcul et la mesure est très satisfaisant.

\section{CONCLUSION}

Nous avons présenté différentes possibilités d'utilisation de la réflectivité de neutrons polarisés pour l'étude de films minces. Les études étaient limitées à la caractérisation de profils magnétiques en profondeur dans les multicouches. Il est possible d'étendre ces études pour caractériser les structures planaires dans des syst èmes multicouches. Cela sera présenté dans le chapitre suivant qui discute de la diffusion hors spéculaire et de la diffusion en incidence rasante (Grazing Incidence SANS). 